\title{
LAS FORMAS DE ORGANIZACIÓN Y ASOCIACIÓN DE LOS TRABAJADORES DE EMPRESAS RECUPERADAS EN LA ARGENTINA (2000-2015).
}

THE FORMS OF ORGANIZATION AND ASSOCIATION OF RECUPERATED ENTERPRISES' WORKERS IN ARGENTINA (2000-2015).

LES FORMES D'ORGANISATION ET D'ASSOCIATION DES TRAVAILLEURS DES ENTREPRISES RECUPEREES EN ARGENTINE (2000-2015).

AS FORMAS DE ORGANIZAÇÃO E ASSOCIAÇÃO DOS TRABALHADORES DE EMPRESAS RECUPERADAS NA ARGENTINA (2000-2015).

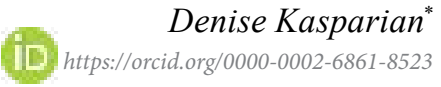

RESUMEN: En términos generales, las empresas recuperadas se basan en el trabajo asociativo y la propiedad conjunta de los medios de producción, por lo cual constituyen formas productivas no estructuradas a partir de relaciones asalariadas, en las cuales el trabajo se organiza a partir de patrones más horizontales y flexibles de relacionamiento, y la participación y la subjetividad de los trabajadores adquieren relevancia. El objetivo de este artículo es describir las formas de organización y asociación supra-unidad productiva que estos trabajadores sin patrón integraron y/o conformaron para la consecución de una multiplicidad de objetivos. El diseño metodológico es de corte cualitativo y triangula una serie de técnicas de investigación: entrevistas a informantes clave, análisis documental y de bibliografía sobre la temática, y búsqueda sistemática y análisis de artículos periodísticos sobre las acciones colectivas llevadas a cabo por organizaciones vinculadas al proceso de recuperación de empresas. Se observa que mientras que en un primer momento estos trabajadores crean organizaciones sociales que conforman un movimiento social, con la estabilización del proceso, dicho movimiento se fragmenta y da lugar a una convergencia cooperativa en organizaciones de segundo y tercer grado, al acercamiento sindical, y a la conformación de redes sectoriales. Hacia el final del período, se

\footnotetext{
* Doutora en Ciencias Sociales, Instituto de Investigaciones Gino Germani, Facultad de Ciencias Sociales, Universidad de Buenos Aires (UBA), Buenos Aires, Argentina; e-mail: denise.kasparian@gmail.com
} 
observa una incipiente revitalización del carácter movimentista de las organizaciones de empresas recuperadas.

Palabras clave: Empresas recuperadas; Cooperativas; Organizaciones sociales; Trabajadores no asalariados; Acción colectiva.

ABSTRACT: In general terms, worker-recuperated enterprises are based on associative work and joint ownership of the means of production, which is why they constitute productive forms not based on salaried relationships. In these enterprises, work is organized upon more horizontal and flexible patterns, and workers' participation and subjectivity acquire relevance. The objective of this article is to describe the forms of organization and association that these workers without boss, at a supra-productive unit level, integrate and/or create to achieve a multiplicity of objectives. The methodological design is of a qualitative type and triangulates a series of research techniques: interviews with key informants, analysis of documents and bibliography, and systematic research and analysis of journalistic articles on collective actions carried out by organizations linked to the process of worker-recuperated enterprises. It is observed that while, at first, these workers create social organizations that integrate a social movement, with the stabilization of the process, the movement tends to fragment and leads to cooperative convergence in organizations of second and third degree, union approach, and the formation of cluster networks. Towards the end of the period, an incipient revitalization of the worker-recuperated enterprises' movement is observed.

Keywords: Worker-recuperated enterprises; Cooperatives; Social organizations; Non-salaried workers; Collective action.

$\boldsymbol{R} \boldsymbol{E} \boldsymbol{S U M E}:$ En générale, les entreprises récupérées sont basées sur le travail associatif et la propriété commune des moyens de production, donc elles constituent des formes productives non structurées sur des relations salariées. Alors, le travail est organisé à partir de modèles plus horizontaux et des relations flexibles, et la participation et la subjectivité des travailleurs acquièrent de la pertinence. L'objectif de cet article est de décrire les formes d'organisation et d'association supra-unité productive que ces travailleurs sans patron intègrent et/ou forment pour atteindre une multiplicité d'objectifs. Le dessin méthodologique est qualitative et triangule une série de techniques de recherche: des entretiens avec des informateurs clés, l'analyse des documents et de la littérature sur le sujet, et la recherche systématique et l'analyse des articles de journaux sur les actions collectives menées par les organisations impliquées 
Las formas de organización y asociación de los trabajadores de empresas recuperadas en la Argentina (2000-2015).

dans le processus de la récupération d'entreprises. On observe que, d'abord, les travailleurs s'organisent à partir de la création d'organisations sociales qui conforment un mouvement social, mais, avec la stabilisation du processus, le mouvement social se fragmente et l'on observe une convergence coopérative dans des organisations de deuxième et troisième degré, une approche syndicale, et la formation de réseaux sectoriels. Vers la fin de la période, on observe une revitalisation de la nature mouvementiste des organisations d'entreprises récupérées.

Mots-clés: Entreprises récupérées; Coopératives; Organisations sociales; Travailleurs non-salariés; Action collective.

RESUMO: De modo geral, as empresas recuperadas funcionam com base no trabalho associativo e na propriedade conjunta dos meios de produção, constituindo formas produtivas não estruturadas sobre relações assalariadas, em que o trabalho é organizado a partir de padrões mais horizontais e relações flexíveis, e a participação e subjetividade dos trabalhadores adquirem relevância. $O$ objetivo deste artigo é descrever as formas de organização e associação que estes trabalhadores sem chefe integraram el ou formaram por encima das unidades de produção para alcançar uma multiplicidade de objetivos. $O$ desenho metodológico é qualitativo e triangula uma série de técnicas de pesquisa: entrevistas com informantes-chave, análise de documentos e literatura sobre a temática, assim como uma análise sistemática de artigos de jornal sobre as ações coletivas empreendidas por organizações ligadas ao processo de recuperação de empresas. Observa-se que, si inicialmente estes trabalhadores criam organizações sociais que compõem um movimento social, com a estabilização do processo, o movimento fragmenta-se, e observa-se uma convergência cooperativa em organizações de segundo e terceiro grau, una aproximação sindical, e uma formação de redes setoriais. Ao final do periodo, observa-se uma revitalização incipiente do caráter movimentista das organizações de empresas recuperadas.

Palavras chave: Empresas recuperadas; Cooperativas; Organizações sociais; Trabalhadores não assalariados; Ação coletiva.

\section{INTRODUCCIÓN}

En la Argentina, las empresas recuperadas refieren a unidades socioproductivas gestionadas por sus trabajadores, que se originan en la reconversión de empresas capitalistas en crisis. Frente a la vul- 
neración de la relación salarial por parte del capital, los trabajadores, en la defensa de su puesto laboral, avanzan sobre la dirección de la producción, conformando generalmente cooperativas de trabajo.

Si bien pueden rastrearse experiencias previas con características similares, fue a partir de la crisis generalizada de los años 2001-2002 que este fenómeno se difundió súbitamente (Palomino, 2005; Rebón, 2007; Salgado, 2012). Según los últimos datos disponibles, a fines del año 2017 eran 368 las empresas recuperadas que atravesaban distintas ramas productivas y ocupaban a más de 15 mil trabajadores (Programa Facultad Abierta, 2017). Alrededor de la mitad de este universo se concentraba en el Área Metropolitana de Buenos Aires, no obstante lo cual, se registraba presencia de estas unidades productivas en 20 de los 24 distritos de la Argentina (Programa Facultad Abierta, 2017).

En términos generales, las empresas recuperadas se basan en el trabajo asociativo y la propiedad conjunta de los medios de producción, por lo cual configuran un modo de organizar la producción alternativo al capitalismo. En este sentido, constituyen formas productivas no estructuradas a partir de relaciones asalariadas, en las cuales el trabajo se organiza a partir de patrones más horizontales y flexibles de relacionamiento, y la participación y la subjetividad de los trabajadores adquieren relevancia (Kasparian, 2017). En otras palabras, y tal como han señalado otros investigadores (Fajn, 2003; Rebón, 2007; Salgado, 2012), la desobediencia de las heteronomías clásicas del ámbito fabril y la recuperación de la empresa dan lugar al aumento de los márgenes de autodeterminación en el ámbito de trabajo.

Sin embargo, el pasaje del trabajo asalariado al trabajo asociativo y autogestionado en las empresas recuperadas puede implicar una relativa precarización en términos de los derechos laborales a los que acceden los trabajadores. Respecto a la protección social, ante la falta de un régimen especial para cooperativas de trabajo, las empresas recuperadas suelen acceder a un régimen simplificado destinado a trabajadores autónomos ${ }^{1}$, que incluye impuestos al siste-

1 El acceso a la seguridad social de los trabajadores de cooperativas de trabajo es reglamentado por la resolución 784/92 de la Administración Nacional de la Seguridad Social (ANSES), que en su artículo $1^{\circ}$ establece que, al no tratarse de trabajadores en relación de dependencia, los asociados son considerados autónomos. Además, la resolución 183/92 del Instituto Nacional de Acción Cooperativa (INAC, organismo que luego será el Instituto Nacional de Asociativismo y Economía Social) establece que las cooperativas de trabajo deben garantizar el acceso a la seguridad social de 
ma tributario y aportes al sistema de seguridad social (jubilación y obra social), pero que excluye a los trabajadores de las asignaciones familiares, el seguro de desempleo y el seguro de riesgos de trabajo. Además, este régimen posee la desventaja de que, al momento de la jubilación, sólo permite acceder al haber mínimo. Respecto a la estabilidad laboral, si bien en términos individuales, el carácter de asociado que revisten los trabajadores otorga mayor estabilidad en comparación a la empresa capitalista ${ }^{2}$, muchas recuperadas se encuentran en posiciones de vulnerabilidad debido a la tenencia precaria de los inmuebles en donde funcionan. Ahora bien, más allá de los elementos enumerados, cabe recordar que la recuperación de las empresas suele expresar la reversión del proceso de pauperización social que origina el retiro capitalista de la producción (Salgado, 2012).

Así, en comparación con la paradigmática relación salarial industrial con protección social, estas experiencias podrían ubicarse dentro lo que se ha llamado trabajo no clásico (De la Garza Toledo, 2011). En este sentido, una línea de indagación en la literatura sobre la temática ha girado en torno a las formas de organización y asociación supra-unidad productiva que estos trabajadores sin patrón integraron y/o conformaron para la consecución de una multiplicidad de objetivos (Dávolos y Perelman, 2003; Palomino, 2003, 2005; Palomino, Bleynat, Garro y Giacomuzzi, 2011; Wyczykier, 2009). Entre éstos podemos mencionar, la recuperación de nuevas empresas a partir de la acumulación de experiencias y aprendizajes, la sanción de leyes de expropiación para obtener la tenencia de los bienes muebles e inmuebles en donde funcionan las cooperativas, la representación gremial del sector para lograr el desarrollo de políticas públicas, el planteo de demandas a los distintos poderes y niveles estatales a través de acciones colectivas, la compra o comercialización conjunta, y la provisión de asesoría

sus asociados, cumpliendo con las aportaciones necesarias a los fines del régimen previsional en el sistema de trabajadores autónomos (Deux Marzi y Hintze, 2014). A fines del año 2013, el Instituto Nacional de Asociativismo y Economía Social (INAES) dictó la resolución 4.664/13, que reemplaza la 183/92. La misma introduce dos novedades respecto a la seguridad social de trabajadores de cooperativas de trabajo: 1) la posibilidad de optar entre la realización de aportes al régimen autónomo (monotributo) o al régimen en relación de dependencia; 2) insta a las Aseguradoras de Riesgo de Trabajo (ART) a emitir sus pólizas a favor de las cooperativas. Respecto a este último punto, las cooperativas enfrentan fuertes dificultades en tanto la ley referida a las ART estipula requisitos que las cooperativas no pueden cumplir.

2 El artículo $23^{\circ}$ de la Ley de Cooperativas $\mathrm{N}^{\circ} 20.337$ establece que todos los asociados tienen derecho a apelar su exclusión ante la asamblea de asociados. 
legal, técnica y política. Otros estudios, sin profundizar en el análisis específico de las empresas recuperadas, enfatizaron en la necesidad de que los emprendimientos asociativos y autogestionados se inserten en redes de colaboración y apoyo mutuo, integrando procesos más amplios de transformación económica, política y cultural, que permitan la sostenibilidad colectiva del sector (Sousa Santos y Rodríguez, 2011; Vázquez, 2014; Williams, 2014; Wright, 2015).

Siguiendo los trazos marcados por la literatura existente, el objetivo de este artículo es describir las formas de organización y asociación supra-unidad productiva de los trabajadores de empresas recuperadas en el período 2000-2015 en la Argentina. El diseño metodológico es de corte cualitativo y triangula una serie de técnicas de investigación. Recurrimos a entrevistas a informantes clave, al análisis documental y de bibliografía sobre la temática, y a la búsqueda sistemática y análisis de artículos periodísticos sobre las acciones colectivas llevadas a cabo por organizaciones de empresas recuperadas.

A continuación, describimos estas formas de organización y asociación a partir de una periodización que identifica dos momentos: la sociogénesis del proceso y su estabilización. Finalmente, esbozamos unas reflexiones finales de cara a los desafíos que instaura la actualidad política, social y económica en la Argentina y en la región para la organización de los trabajadores sin patrón.

\section{EI movimiento de empresas recuperadas en la sociogénesis del proceso}

Si bien la recuperación de empresas representa un proceso novedoso anclado en la crisis generalizada de los años 2001-2002 ${ }^{3}$, no puede perderse de vista que se encuentra anudada a tradiciones de lucha y de organización de las clases trabajadoras argentinas. Retomando la toma de fábricas y la modalidad autogestionada de organización desarrollada a principios de la década del ochenta en respues-

3 Entre fines del siglo XX y comienzos del siglo XXI en la Argentina tuvo lugar la consolidación y la crisis del modelo de acumulación basado en la valorización financiera (Basualdo, 2013). Este modelo, que había comenzado a configurarse a partir de la dictadura cívico-militar de los años 1976-1983, se basaba en la especulación financiera sostenida en el endeudamiento externo y beneficiada por el diferencial de tasas de interés nacionales e internacionales. Una de sus consecuencias fue la desindustrialización de la economía argentina. La crisis generalizada de los años 2001-2002 trajo aparejada la eclosión y crisis estructural de dicho modelo económico, y también el cuestionamiento y la crisis de diversas dimensiones referentes a la política y la cultura. 
ta a los primeros indicios de desindustrialización (Palomino, 2003), la recuperación de empresas puede considerarse como un capítulo de la lucha de las clases trabajadoras argentinas en un contexto de crisis generalizada, y en el marco de las profundas transformaciones del repertorio de la protesta laboral que tuvo lugar con la instauración democrática en el año 1983 y con mayor intensidad a partir de la década del noventa (Farinetti, 1999) ${ }^{4}$. Constituyéndose en una nueva herramienta de presión y negociación con el capital, la recuperación de empresas incidió incluso sobre el sistema institucionalizado de relaciones laborales (Palomino, 2003).

La ruptura con el marco precedente de la lucha de las clases trabajadoras argentinas radicó, principalmente, en que los sindicatos no tuvieron un rol de importancia en las primeras recuperaciones que se desencadenaron a partir de la crisis generalizada. En los primeros momentos, el rol de los sindicatos no fue uniforme. Mientras que algunos participaron activamente en apoyo a los trabajadores, acompañando los conflictos anteriores, así como el proceso de recuperación, otros adoptaron una actitud que osciló entre la pasividad y la oposición a la recuperación, y finalmente, otro grupo llegó a actuar en connivencia con la patronal (Dávolos y Perelman, 2003; Palomino, 2005). Así, salvo algunas excepciones ${ }^{5}$, los trabajadores que emprendían la recuperación enfrentaron la indiferencia y el abandono de los sindicalistas. En el contexto de los conflictos que se suscitaban en las empresas en crisis y en los cuales se iba configurando la opción de la recuperación, la pérdida del rol dirigencial por parte de los sindicatos dio lugar a una autonomización de los colectivos de trabajadores respecto de aquellos. De este modo, la carencia de una forma organizativa que proporcionara a los trabajadores orientación estratégica y soluciones frente a la amenaza de la pérdida de la fuente laboral promovió la constitución de los denominados movimientos de empresas recuperadas (Palomino, 2005; Rebón, 2007).

4 Farinetti (1999) sostiene que las nuevas formas de protesta laboral (los estallidos sociales y los cortes de ruta) se diferencian del repertorio clásico, asociado al sindicalismo de raigambre peronista. En las nuevas formas, los reclamos se dirigen a la satisfacción de necesidades básicas, son más puntuales y más defensivos que los contenidos en las formas clásicas, y las acciones están menos institucionalizadas y son más espontáneas.

5 En la sociogénesis del proceso de recuperación de empresas, las principales excepciones fueron la seccional Quilmes de la Unión Obrera Metalúrgica, la Federación Gráfica Bonaerense, y el Sindicato Ceramista de Neuquén. 
Esto permitió que la recuperación de empresas se constituyera en un recurso disponible más allá del apoyo sindical con el que contaran las unidades productivas (Dávolos y Perelman, 2003). En este sentido, Wyczykier (2009) utilizó la noción de recolectivización para referirse a la reconstrucción de protecciones colectivas perdidas y/o fragilizadas en los últimos lustros del siglo XX que implicó el proceso de recuperación de empresas y la consiguiente conformación de colectivos protectores que configuraron espacios de representación de intereses y de recreación de vínculos identitarios a partir de nuevas solidaridades intergrupales.

Mientras que las primeras recuperaciones se dieron de forma relativamente aislada, con el trascurrir de las mismas y frente al agravamiento de la situación económica, social y política, las confrontaciones dejaron de reducirse a conflictos entre el patrón y los asalariados de empresas particulares, para contener un amplio abanico de relaciones sociales en las cuales jueces, abogados, funcionarios del Estado, legisladores, miembros de asambleas barriales, universitarios, trabajadores de otras empresas recuperadas, y militantes de partidos políticos, entre otros actores, se hicieron presente, promoviendo y motorizando las recuperaciones.

En resumidas cuentas, la estrategia de recuperación no nació espontáneamente de los trabajadores, sino de su articulación con otros actores que oficiaron de promotores. Éstos se constituyeron en un vector determinante de las recuperaciones; fue su creatividad y determinación lo que posibilitó el avance sobre la dirección de la producción. La interacción entre promotores y trabajadores, junto al conflicto y negociación con otros actores, dio lugar a la conformación de diversas organizaciones de empresas y fábricas recuperadas (Rebón, 2007). Repasemos cuáles fueron las organizaciones que se conformaron en este primer momento de sociogénesis del proceso.

En el año 2000 se formó el Movimiento por la Economía Social (MOPES) a partir de una iniciativa estatal del Instituto Nacional de Asociativismo y Economía Social (INAES) ${ }^{6}$. Este primer intento evolucionó hacia organizaciones que a la vez que daban cuenta

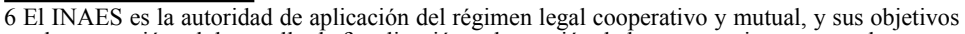
son la promoción, el desarrollo, la fiscalización, y la sanción de las cooperativas y mutuales. 
de la especificidad de los procesos de recuperación de empresas en el marco de la economía social, reflejaban su fragmentación. Así, a pesar de los acuerdos, las diferencias prevalecieron y se confrontaron dos posiciones. Un grupo, liderado por la empresa recuperada IMPA, proponía la conformación de una organización de empresas recuperadas que integrara a todas ellas más allá de la forma jurídica que poseyesen, esto es, sin importar si eran cooperativas, sociedades comerciales clásicas, o si aún no habían definido esta cuestión. Otro grupo, representado por la empresa recuperada Yaguané y la Federación de Cooperativas de Trabajo (FECOOTRA), organización creada en el año 1988 -esto es, con anterioridad a la difusión del proceso de recuperación de empresas-, proponía un agrupamiento exclusivamente por forma jurídica, de modo que las nuevas cooperativas fruto de la recuperación de empresas se integraran a FECOOTRA, dejando de lado a las experiencias de recuperación que no optaran por conformar cooperativas de trabajo (Rebón, 2007). Antes de proseguir con la caracterización de las organizaciones que finalmente conformó cada agrupamiento, revisemos la cuestión de la forma jurídica que adoptaron las empresas recuperadas.

La literatura señala que las empresas recuperadas constituyen experiencias de cooperativismo adaptativo, dado que lejos de asumir una vocación cooperativista fundada doctrinariamente, los trabajadores encontraron en la forma organizativa y jurídica de la cooperativa de trabajo la modalidad más adecuada y efectiva para defender sus puestos laborales (Palomino, 2003; Rebón, 2007). Este encuadre jurídico se correspondía con las necesidades y las prácticas de los colectivos de trabajadores que emprendían la autogestión, a la vez que afianzaba la legalidad de las experiencias (Echaide, 2004, 2010). Además, poseía las ventajas de la exención del pago del impuesto a las ganancias y las deudas de la empresa anterior (Palomino, 2003). En los comienzos de la difusión de estos procesos, la forma jurídica cooperativa de trabajo convivió con otras estrategias, entre las que destacaba la estatización con control obrero. Esta propuesta consistía en que el Estado expropiara la empresa y la pusiera bajo el control y la administración de los trabajadores, garantizando a la vez sus condiciones laborales. De este modo, se buscaba establecer formas de 
propiedad social y colocar la autogestión de los trabajadores al servicio de una actividad productiva acorde a las necesidades de la sociedad. Este modelo fue impulsado por partidos políticos y sectores de izquierda, y sostenido en sus primeros momentos por algunas pocas empresas recuperadas. El debilitamiento de esta estrategia frente a la de conformar cooperativas para luego buscar la expropiación de los bienes muebles e inmuebles de las empresas residió en que la estatización no contemplaba la necesidad de constituir una cobertura legal transitoria (Rebón, 2007). De este modo, la conformación de cooperativas y la lucha por la expropiación con instancias intermedias de arreglos judiciales terminó por imponerse ${ }^{7}$. Ahora sí, retomemos la descripción de las organizaciones.

En el año 2001, el primer grupo liderado por la empresa IMPA fundó el Movimiento Nacional de Empresas Recuperadas (MNER), organización que se constituyó en una de las de mayor injerencia. El lema que sintetiza la estrategia del movimiento es "Ocupar, resistir y producir”. Esta organización se caracteriza por no poseer una estructura jerárquica y orgánica, sino que más bien se nutre de la militancia de algunos referentes (Rebón, 2007). Respecto del segundo grupo, por su fuerte arraigo al ideario cooperativista y su rechazo a la conformación de cooperativas como estrategia adaptativa, FECOOTRA no tuvo una participación directa en las recuperaciones y prosiguió su camino como federación de cooperativas. Por último, como un desprendimiento de FECOOTRA, Yaguané conformó la Federación Nacional de Cooperativas de Trabajo y Empresas Reconvertidas (FENCOOTER) (Rebón, 2007), la cual no llegó a tener una gran ascendencia en las empresas recuperadas.

En el contexto de sociogénesis del proceso de recuperación de empresas, esto es, entre los años 2001 y 2003 aproximadamente, las empresas recuperadas y sus organizaciones trascendieron la forma de agregación clásica de las clases trabajadoras -el sindicato-, conformando un movimiento social. Siguiendo a Tilly y Wood (2010), definimos a los movimientos sociales como una forma de contienda política que desarrolla, con cierta permanencia en el tiempo, una

7 Ya en el año 2004, el $94 \%$ de las empresas recuperadas existentes habían adquirido la forma jurídica cooperativa de trabajo (Ruggeri, 2010). 
campaña pública de planteo de un conjunto articulado de reivindicaciones colectivas a las autoridades estatales -sean éstas objeto de las reivindicaciones, aliados o árbitros de la lucha- a través de un repertorio delimitado de acciones colectivas. La noción de contienda refiere a que los movimientos sociales se caracterizan por plantear reivindicaciones que chocan con los intereses de otros grupos. Por su parte, el carácter político de dicha contienda señala su dimensión institucional, en tanto los movimientos interpelan a los gobiernos. En dicha campaña, además, los movimientos sociales realizan manifestaciones públicas de su valor, unidad, peso numérico y compromiso. En este sentido, el movimiento de empresas recuperadas logró plantear en la escena pública y frente a las autoridades estatales, la recuperación (que de modo paradigmático implica la ocupación del establecimiento productivo) como forma de acción en defensa de las fuentes de trabajo. Si bien el movimiento encontró su núcleo en los trabajadores de empresas recuperadas y en la demanda por la fuente de trabajo, fue producto de la articulación de una embrionaria alianza social que trascendió a las identidades presentes en las empresas.

Este período que denominamos de carácter movimentista albergó grandes eventos de acción colectiva protagonizados por el movimiento de empresas recuperadas. A modo ilustrativo, traemos dos ejemplos para dar cuenta no sólo de la magnitud de los eventos, sino también de las articulaciones sociales que los sostuvieron. En primer lugar, cabe mencionar la masiva movilización que tuvo lugar el 22 de abril de 2003 en la Ciudad de Buenos Aires para enfrentar el desalojo de una empresa recuperada de la industria textil. Al menos 5 mil personas se movilizaron aquella jornada desde el Congreso de la Nación hasta la fábrica situada en el barrio de Balvanera (Brukman: falló una gestión oficial y las obreras acampan en la calle, 23 de abril de 2003). Las trabajadoras textiles fueron acompañadas por más de 30 organizaciones, entre las cuales no sólo se encontraban organizaciones de empresas recuperadas, sino también partidos políticos de izquierda y organizaciones de trabajadores desocupados.

En segundo lugar, el 11 de junio de 2003 tuvo lugar una masiva movilización (participaron alrededor de 10 mil personas), en la cual el movimiento de empresas recuperadas confluyó con orga- 
nizaciones de trabajadores desocupados, así como de jubilados y pensionados, en una jornada nacional por el trabajo y el salario. En dicha oportunidad, las demandas de las empresas recuperadas (expropiación de todas las empresas recuperadas, asignación de capital de trabajo, créditos accesibles a través de la banca pública, salida de la policía de las empresas recuperadas, y apoyo a las mismas) ocuparon un lugar destacado (Jornada por el salario y el trabajo. Fotos, 11 de junio de 2003).

En términos generales, el movimiento tendió a recurrir a la acción colectiva frente a nuevos casos de recuperación de empresas, para resistir desalojos y sostener ocupaciones de empresas en marcha, así como para obtener leyes de expropiación. Su repertorio se asentó sobre dos formas de acción. Por un lado, para la recuperación de las empresas y en oposición al ex empleador, los colectivos laborales tendieron a ocupar los establecimientos productivos con la ayuda de promotores y demás identidades sociales mencionadas anteriormente. Por otro lado, recurrieron a la movilización como modo de plantear sus demandas a las autoridades estatales en torno a los casos emblemáticos y más conflictivos, y para el planteo de demandas generales en torno a la regulación de todas las empresas recuperadas, como la expropiación de todas las fábricas, el otorgamiento de capital de trabajo y el desarrollo de líneas crediticias.

Por último, cabe aclarar que, si bien tuvieron lugar los eventos descriptos, el movimiento se caracterizó por poseer una baja penetración al interior de las empresas, asentándose principalmente sobre el activismo de algunos referentes y dirigentes, y por su baja capacidad de movilización. Esto se encuentra asociado con la necesidad de poner en marcha la producción y con las dificultades que plantea en este sentido la participación del pleno del colectivo laboral en las acciones colectivas:

Eso es un problema y es clave. Que tampoco está resuelto, porque yo estoy jubilado, voy, vengo, ahora me voy a otra reunión, lo hago porque me gusta, porque quiero. Cuando acá tienen producción, moverlos es difícil. [El compañero] si hay mucho trabajo a veces no se puede ir. No porque se lo prohíba, sino porque hay una cuestión 
Las formas de organización y asociación de los trabajadores de empresas recuperadas en la Argentina (2000-2015).

de cumplir con el trabajo. (...) Cuando se empezó a formar el Movimiento de Empresas Recuperadas en IMPA, íbamos otro compañero y yo que también se jubiló, íbamos los sábados, pero lo hacíamos porque nos gustaba a nosotros. Y cuando veníamos acá no pasaba nada, no nos daba pelota nadie. Íbamos al movimiento de empresas recuperadas, le contábamos al Consejo y nada. Y cuando venías con un problema, acá había 10 problemas más graves entonces lo tuyo quedaba aplacado. Esas son situaciones que se dan. Por eso siempre fue muy difícil movilizar. (...) Cuando empieza un conflicto en una empresa, los compañeros están todos movilizados, pero después, una vez que arranca a funcionar, es mucho más difícil. No podría ser nunca dejar la producción teniendo que entregar a los dos o tres días de trabajo para ir a una movilización. Entonces te mandan unos cuantos de acá, unos cuantos de allá, pero es difícil. (Referente del movimiento de empresas recuperadas, septiembre de 2016)

En el próximo apartado observaremos que a partir de los años 2003-2004 el movimiento comenzó a fragmentarse cada vez más en organizaciones aisladas, teniendo lugar una progresiva pérdida del carácter movimentista del proceso, es decir, las organizaciones tendieron a desarticular sus demandas, así como su planteo a través de acciones colectivas. En un nuevo contexto social, político y económico ${ }^{8}$, las organizaciones diferenciaron sus demandas, $\mathrm{y}$, sobre todo, los modos de canalizarlas y plantearlas a las autoridades estatales.

\section{Fragmentación del movimiento, convergencia cooperativa, acerca- miento sindical y redes sectoriales en la estabilización del proceso}

Tal como mencionamos anteriormente, el carácter movimentista de las organizaciones de empresas recuperadas se fue diluyendo una vez atravesado el momento de sociogénesis del proceso. Esto significa que el conjunto de organizaciones ya no podrá ser caracterizado como un movimiento social, entendido como una forma de

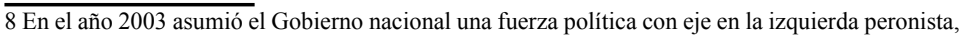
denominada Frente para la Victoria. Gobernó durante el período 2003- 2015 y se caracterizó por producir rupturas sociales, culturales, económicas y políticas respecto a los gobiernos de las dos décadas anteriores. A partir del año 2003, se recuperaron los niveles de empleo y los salarios, y se redujeron los niveles de precariedad laboral (Groisman, 2008). Durante el lustro 2003-2007 tuvo lugar un crecimiento promedio del PBI próximo al $9 \%$ anual, se mantuvieron los superávits fiscal y externo, subieron marcadamente los salarios reales y la ocupación, y la distribución del ingreso mejoró de manera apreciable. 
contienda política con una articulación de demandas planteadas en el espacio público a través de acciones colectivas. Las confluencias de las organizaciones en grandes movilizaciones, como la ejemplificada en el caso de la empresa textil, fueron cediendo terreno frente a la configuración de diversas concepciones acerca de cómo tenían que organizarse las empresas una vez recuperadas, y de disímiles posicionamientos respecto al Gobierno nacional -que por momentos se mostró abierto y receptivo a la recuperación de empresas pero que no por eso institucionalizó completamente el proceso- y, por lo tanto, a las formas de acción adecuadas para plantear las demandas. Estas bifurcaciones, junto a las tensiones producto de la fuerte injerencia de los referentes en las organizaciones, provocaron escisiones en las organizaciones originarias y la creación de nuevas estructuras.

La primera fractura del MNER tuvo lugar en el año 2003, a sólo dos años de su creación, a partir de la conformación del Movimiento Nacional de Fábricas Recuperadas por los Trabajadores (MNFRT). A diferencia del MNER, su estrategia ha sido más legalista, razón por la cual se focaliza en institucionalizar el proceso a través de reformas legislativas $\mathrm{y}$, a pesar de que diversos proyectos de modificación de la Ley de Concursos y Quiebras fueron elaborados por distintos espacios sociales y políticos, fue su referente quien logró colocar el proyecto que finalmente culminó en la modificación de la Ley en el año $2011^{9}$. Hacia el año 2013 nucleaba aproximadamente 60 empresas, es decir, alrededor de un quinto de las empresas recuperadas existentes en aquel entonces (Movimiento Nacional de Fábricas Recuperadas por sus trabajadores, s/d). Esta organización es la única que ha permanecido en la postura de aglutinar solamente empresas recuperadas.

La segunda escisión del MNER se produjo en diciembre de 2006 con la creación de una organización de segundo grado ${ }^{10}$, la Fe-

9 Entre las principales modificaciones de la Ley, destacan la posibilidad de que los trabajadores organizados en una cooperativa -incluso en formación- obtengan la continuidad inmediata de la producción de la empresa, la no suspensión de los intereses sobre los sueldos adeudados, y la prioridad para ofertar y acceder a la adjudicación directa de los bienes, utilizando sus créditos laborales. Por lo tanto, la Ley posibilita que los trabajadores sean un actor preponderante frente a un proceso de eventual quiebra, garantizando la preservación de los puestos de trabajo y el funcionamiento de la empresa. Esta reforma de la Ley de Concursos y Quiebras habilita una nueva vía de institucionalización del proceso, estableciendo que ante la quiebra de una empresa los trabajadores conformados en cooperativas pueden utilizar las acreencias laborales para su compra.

10 Las asociaciones de segundo grado se forman por la integración de personas jurídicas, en este caso, de las unidades productivas conformadas en cooperativas de trabajo (asociaciones de primer grado) y se denominan federaciones, mientras que las de tercer grado agrupan tanto a las unidades 
Las formas de organización y asociación de los trabajadores de empresas recuperadas en la Argentina (2000-2015).

deración Argentina de Cooperativas de Trabajadores Autogestionados (FACTA), por parte de uno de los grupos disidentes. Esta escisión se dio como consecuencia de las acusaciones cruzadas de personalismos y de los divergentes posicionamientos políticos frente al Gobierno nacional (Palomino et al., 2011). FACTA se desarrolló a partir de una doble vía. Por un lado, luego de una primera etapa de incorporación de empresas recuperadas, comenzó a incorporar cooperativas de trabajo que no provienen de la recuperación de empresas, en un claro acercamiento al movimiento cooperativo, que terminó de cristalizarse con su incorporación a la Confederación Nacional de Cooperativas de Trabajo (CNCT). La CNCT es una organización de tercer grado constituida en 2009 que agrupa a más de 25 federaciones de cooperativas de trabajo de toda la Argentina y expresa la vinculación entre las empresas recuperadas, las federaciones del cooperativismo tradicional, como FECOOTRA, y las cooperativas vinculadas a la economía social y a la economía popular, como la Federación de Cooperativas de Trabajo Unidas de Florencio Varela (FECOOTRAUN). Esta última federación aglutina cooperativas surgidas a partir del Programa Argentina Trabaja del Ministerio de Desarrollo Social de la Nación ${ }^{11}$. Por otro lado, en el año 2014 formalizó su incorporación a una organización sindical, la Central de Trabajadores de la Argentina (CTA) ${ }^{12}$. En la actualidad, FACTA contiene más de 60 cooperativas conformadas por alrededor de 2.500 trabajadores (Federación Argentina de Cooperativas de Trabajadores Autogestionados, s/d).

Hacia el año 2005, se creó la Asociación Nacional de Trabajadores Autogestionados (ANTA), perteneciente a la CTA. Esta orga-

productivas como a las asociaciones de segundo grado y se denominan confederaciones.

11 El Programa creó cooperativas de trabajo conformadas por población vulnerable para el desarrollo de actividades de infraestructura y mantenimiento de espacios públicos. Los asociados recibían un subsidio individual aportado por el Estado nacional que llegó a representar el $85 \%$ del salario mínimo, vital y móvil. Además, se destinaban fondos para la adquisición de insumos y herramientas. Hacia el año 2015 eran 218.870 los beneficiarios y 5.142 las cooperativas conformadas en el marco del Programa (Ministerio de Desarrollo Social, 2015). En relación al presupuesto, entre los años 2010 y 2013, el Programa contó con el segundo mayor porcentaje del gasto del Ministerio de Desarrollo Social (Arcidiácono y Bermúdez, 2015). Respecto a su expansión territorial, el Programa comenzó a aplicarse en el Gran Buenos Aires y, gradualmente, fue incorporando beneficiarios de 15 de los 24 distritos del país. Con la asunción de una nueva fuerza política al gobierno del Estado nacional a fines del año 2015, el Programa fue perdiendo componentes vinculados al cooperativismo, hasta que en febrero de 2018 fue disuelto.

12 La Central de Trabajadores de la Argentina se creó en el año 1992 y se caracteriza por el trazado de relaciones con organizaciones no ligadas tradicionalmente el mundo del trabajo y por el reclutamiento de afiliados a partir de una definición ampliada de la clase trabajadora. De este modo, posee una lógica cercana al "sindicalismo de movimiento social" (Senén González y Haidar, 2009). 
nización se sustenta principalmente en la experiencia de la empresa recuperada Unión Solidaria de Trabajadores (UST). A diferencia de las demás, ANTA es un sindicato en el cual la pertenencia puede darse en términos individuales, a partir de la afiliación de cada trabajador. De este modo, observamos que mientras que las primeras organizaciones que conformaron las empresas recuperadas tenían un carácter informal de vinculación, con el paso del tiempo, la pertenencia se fue formalizando. Las federaciones y confederaciones requieren adscripciones formales de las cooperativas, mientras que ANTA en tanto sindicato, requiere la afiliación individual de trabajadores pertenecientes a organizaciones económicas autogestionadas sin importar su forma jurídica, incluyendo de este modo a empresas recuperadas, cooperativas y mutuales autogestionadas por sus asociados, emprendimientos de grupos comunitarios, organizaciones sociales y movimientos campesinos, emprendimientos familiares, y de trabajo directo, urbanos y rurales (Asociación Nacional de Trabajadores Autogestionados, s/d).

Finalmente, en el año 2010 surgió otra organización: la Unión Productiva de Empresas Autogestionadas (UPEA). Ésta trasciende, al igual que FACTA y ANTA, el universo de empresas recuperadas. Conformada por 25 empresas recuperadas, cooperativas de trabajo en general y emprendimientos productivos de menor escala, UPEA se planteó como una organización cuyo eje transversal es la autogestión -y no la forma jurídica de organización como en el caso de FACTA-, y su objetivo central, equiparar los derechos del trabajador autogestionado con los del asalariado a partir del establecimiento de un marco jurídico y político adecuado. Así, su principal demanda fue el establecimiento del Estatuto del Trabajador Autogestionado. Una de las características salientes de este agrupamiento fue su acercamiento al Gobierno nacional y, más específicamente, al Ministerio de Trabajo, Empleo y Seguridad Social:

¿Por qué somos cooperativas de trabajo? Porque había una ley de quiebras que decía que sólo se podía conseguir la continuidad de la empresa constituyéndonos en cooperativa de trabajo. La cooperativa de trabajo para nosotros es un estado civil con el cual pode- 
mos seguir intentando subsistir. Muchos compañeros en todos estos años hemos ido recuperando trabajo, recuperando maquinaria, pero tenemos muchas dificultades para el acceso a derechos que tienen los trabajadores plenos. ¿Quiénes son los trabajadores plenos? Los trabajadores que tienen un marco legal, un marco político que los proteja. En el caso de los trabajadores autogestionados de las recuperadas, venimos de empresas en las cuales teníamos un estatuto sindical, jubilaciones, vacaciones, asignación familiar. (...) Lo que les quiero decir es que los que conformamos la UPEA tenemos la convicción de que como trabajadores vamos a seguir pidiendo por un derecho pleno para todos los trabajadores, y para eso pedimos el estatuto de trabajadores autogestionados. (Referente de la UPEA, citado en Kasparian, 2010)

Así, entre las nuevas organizaciones se observa una ampliación de las formas de asociación, salvo en el caso del MNFRT que se mantiene con una lógica de agrupamiento de empresas recuperadas. FACTA se constituye en una federación que opta por un acercamiento al movimiento cooperativo, y al "sindicalismo de movimiento social" (Senén González y Haidar, 2009) a partir del ingreso a la CTA; ANTA da cuenta del desarrollo de un sindicato de trabajadores de organizaciones autogestionadas enmarcado también en la CTA y con una lógica de afiliación individual; y UPEA también amplía el universo a la autogestión y se caracteriza por su relación estrecha con el Ministerio de Trabajo.

Respecto a los eventos de acción colectiva, durante este segundo período no tuvieron lugar acciones de la magnitud de las movilizaciones del período anterior. Si bien la movilización continuó siendo la forma de acción colectiva utilizada para la irrupción en la escena pública, en los años 2006 y 2007 también se desarrollaron algunas ferias de empresas recuperadas, en las cuales se exhibieron los bienes producidos como modo de instalar demandas. Éstas se modificaron respecto al período anteriormente reseñado. Además de las movilizaciones en defensa de casos específicos y emblemáticos, principalmente en torno a la propiedad de los inmuebles, se realizaron movilizaciones demandando la sanción de una ley nacional de expropiación de todas las empresas recuperadas, la modificación de la Ley de Concursos y 
Quiebras, y en contra de los vetos de las leyes de expropiación realizados por el jefe de Gobierno de la Ciudad de Buenos Aires. En este último caso se realizaron conferencias de prensa frente a la Jefatura de Gobierno de forma conjunta entre legisladores de la Ciudad de Buenos Aires y trabajadores de empresas recuperadas.

Además, entre mediados de 2011 y principios de 2012 tuvieron lugar movilizaciones, en algunos casos de carácter nacional, en respuesta a la intención del Gobierno nacional de dar de baja un subsidio destinado a trabajadores de empresas autogestionadas. Este hecho conformó una estructura de oportunidades políticas para la acción colectiva y articulada de diversos actores. El 7 y el 28 de marzo, el Encuentro Amplio de Cooperativas desarrolló jornadas de movilizaciones en las ciudades de Buenos Aires, Rosario, La Rioja, Formosa, Neuquén y Tucumán, en demanda principalmente de la renovación y el aumento de dicha línea de financiamiento. Además, se reclamó una ley de expropiación nacional y políticas de fondo para el sector. Formaron parte del Encuentro Amplio de Cooperativas, empresas recuperadas emblemáticas, ANTA, la Confederación de Trabajadores de la Economía Popular (CTEP), cercana al sindicalismo tradicional de la Confederación General del Trabajo (CGT), y la Asociación Gremial de Trabajadores Cooperativistas Autogestivos y Precarizados (AGTCAP), organización que nuclea a cooperativas de programas sociales, principalmente del Programa Argentina Trabaja.

En esta instancia, resulta pertinente detenernos en la CTEP, por la creciente visibilización que ha ido obteniendo desde su conformación. Esta organización gremial se creó el 17 de agosto de $2011^{13}$ $y$, si bien su origen se asocia a la conformación de cooperativas en el marco del Programa Argentina Trabaja, también retoma otras experiencias de trabajo que se enmarcan en la denominada economía popular. Ésta ha sido definida como las actividades desarrolladas por los sectores populares excluidos del mundo del trabajo asalariado o por aquellos que buscan ingresos complementarios en el trabajo por cuenta propia, con el objetivo de garantizar la satisfacción de las necesidades básicas (Maldován Bonelli, 2018; Sarria Icaza y Tiribia,

13 En su acto fundacional, participó el Movimiento Nacional de Empresas Recuperadas por los Trabajadores (MNER). 
Las formas de organización y asociación de los trabajadores de empresas recuperadas en la Argentina (2000-2015).

2004). En este sentido, agrupa a trabajadores cuentapropistas, autogestionados, cooperativistas y de programas sociales, entre otros.

Esta asociación gremial de representación de los trabajadores de la economía popular parte del diagnóstico de que es necesario restituir los derechos laborales y sociales que los años de neoliberalismo erosionaron. Además, postula que en la economía popular la militancia resulta fundamental para construir unidad y activar las luchas, dada la dispersión de los trabajadores, así como la ausencia de una patronal identificable (Acto fundacional de la Confederación de Trabajadores de la Economía Popular, 19 de agosto de 2011; Argentina. ¿Qué es la CTEP?, s/d; CTEP, s/d). En el año 2015, la CTEP logró que se creara un régimen de agremiación para los trabajadores de la economía popular y el Ministerio de Trabajo le concedió la atribución de representar los intereses colectivos de dichos trabajadores (Reconocimiento para los trabajadores de la economía popular, 14 de diciembre de 2015).

En suma, por un lado, tal como han planteado Palomino et al. (2011), las lógicas originales de asociación centradas en las organizaciones de empresas recuperadas -surgidas de la confluencia entre promotores ajenos a las unidades productivas y trabajadores de las recuperadas- dieron paso a formas más amplias de asociación-federativas, confederativas, entre otras-, permitiendo una articulación mayor con organizaciones del cooperativismo tradicional y de experiencias de autogestión -generalmente conformadas como cooperativas- ligadas a emprendimientos de la economía social y popular. Denominamos a este proceso convergencia cooperativa, en tanto las diversas trayectorias cooperativas fueron confluyendo en organizaciones amplias. Por otro lado, las formas de asociación también dieron cuenta de un acercamiento sindical, más fuerte respecto al "sindicalismo de movimientos sociales", pero también presente en el caso del sindicalismo tradicional, cuya organización emblemática es la GCT.

La instalación cultural del proceso de recuperación de empresas (Rebón, Kasparian y Hernández, 2015), junto con sus repercusiones en el ámbito estatal, fueron elementos fundamentales en la mencionada convergencia. En primer lugar, recordemos que la adopción adaptativa de la forma jurídica cooperativa de trabajo (Palomino, 2003; Rebón, 2007) había sido leída por el cooperativismo tradicio- 
nal de forma negativa, en tanto consideraba que dicha adopción no adscribía a los valores del movimiento cooperativo. Por esta razón, había ocupado un rol secundario durante la sociogénesis del proceso de recuperación de empresas. No obstante, con el correr de los años, la potencia simbólica del movimiento de empresas recuperadas terminaría por promover una reinterpretación de la memoria histórica de este actor que se había mantenido relativamente al margen, estimulando su participación (Palomino et al., 2011). En este sentido son elocuentes las palabras de quien fuera presidente de FECOOTRA, que incluso identifica las primeras empresas recuperadas durante los gobiernos de Juan Domingo Perón:

Es que casi todas las cooperativas de trabajo son recuperadas. La historia del cooperativismo de trabajo empezó en 1918 en el país (...). Después viene el proceso de Perón, ahí se recuperan las primeras industriales. Las primeras son recuperadas. (...) O sea, la mayoría de las cooperativas siempre surgen de una crisis en una empresa y los trabajadores la recuperan. Hoy ya es moneda corriente. Son todas cooperativas de trabajo, lo que pasa es que algunas... La única que yo conozco que surgió por voluntad de los asociados es la mía. (...) Tenés las empresas recuperadas de antes del 2000 y después del 2000. Las de antes del 2000 tienen la característica de que son empresas viejas. (...) cooperativas de trabajo son todas, cada una tiene una historia de conformación diferente. (Autoridad de FECOOTRA, mayo de 2013)

En segundo lugar, también consideramos central en este proceso las políticas públicas que contribuyeron a la conformación del campo de la economía social y, dentro de ésta, del trabajo asociativo y autogestionado ${ }^{14}$. Por el lado de las empresas recuperadas, la necesidad de resolver cuestiones de gestión y el declive de las posturas más radicalizadas contra el cooperativismo (Palomino et al., 2011) fueron elementos que abonaron este cambio en las lógicas de asociación. Así, mientras que en la sociogénesis de los procesos de recuperación de empresas, la adopción de la forma cooperativa de trabajo resultó una estrategia adaptativa, en la actualidad las fronteras entre las empresas recuperadas y las cooperativas tradicionales se tornan difusas.

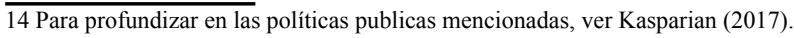


En otro orden de cosas, además de las nuevas organizaciones con lógicas diversas de asociación, se desarrolló un proceso de constitución de redes sectoriales o clusters conformados por cooperativas de trabajo en general, es decir, no sólo empresas recuperadas. Así, a la lógica de asociación política y reivindicativa, se le adicionaron las formas organizativas con fines productivos y comerciales. Los beneficios que otorga la integración sectorial surgen de la implementación de políticas comunes en materias relacionadas con compras, almacenamiento, producción, gestión de calidad, recursos humanos, capacitación y formación, financiamiento, comercialización y difusión (Kasparian y Hernández, 2010). Salvo la Red Gráfica Cooperativa que se conformó en el año 2006, las demás redes se crearon entre los años 2012 y 2013. Entre éstas podemos mencionar, la Red Textil Cooperativa, la Red Metalúrgica Nacional Cooperativa, la Federación de Cooperativas Autogestionadas de la Carne y Afines y la Federación de Organizaciones Productoras de Alimentos. Observamos que estos clusters de integración sectorial suelen expresar también un proceso de convergencia cooperativa y de acercamiento sindical. En este sentido, en la conformación de la Red Gráfica Cooperativa resultó central el rol de la Federación Gráfica Bonaerense, así como en la conformación de la Red Metalúrgica jugaron roles importantes la seccional Quilmes de la Unión Obrera Metalúrgica y FECOOTRA. En el caso de la Red Textil y de la Federación de Organizaciones Productoras de Alimentos, el actor dinamizador fue la CNCT.

Finalmente, hacia fines del período bajo estudio, ANTA y UPEA habían tendido a perder injerencia entre las empresas recuperadas, mientras que FACTA y MNFRT permanecían. Por último, en el año 2015, a partir de una articulación con la CTEP, y en el contexto de las elecciones presidenciales, el MNER, que había perdido peso a raíz de sus múltiples escisiones, irrumpió nuevamente en la escena pública.

En este sentido, promovido por el contexto electoral y la posibilidad del retorno de políticas neoliberales con el cambio de gobierno, en el año 2015, las organizaciones participaron de manera conjunta en actos y movilizaciones. Así, y más allá del período que abarca este artículo, podemos hipotetizar que el cambio de contexto social, político y económico a partir de la asunción de un nuevo 
gobierno en diciembre de 2015 promovió una revitalización de la acción colectiva en el espacio público por parte de las organizaciones de empresas recuperadas, así como una transformación de las demandas, las cuales adquirieron un carácter más defensivo, en el marco de una ampliación de las luchas sindicales de carácter defensivo en la Argentina. A modo ilustrativo, el 20 de julio de 2016 tuvo lugar una movilización que aglutinó a 10 mil trabajadores provenientes de empresas recuperadas, de cooperativas del Programa Argentina Trabaja y de otros programas sociales, junto con organizaciones de PyMEs y de consumidores y usuarios. La movilización tuvo como finalidad demandar una tarifa social en los servicios básicos (electricidad y gas) para las cooperativas de trabajo frente a la fuerte suba de las tarifas. El contexto y las demandas se habían modificado.

\section{REFLEXIONES FINALES}

Guiados por el objetivo de indagar en las formas de organización y asociación de los trabajadores sin patrón en la actualidad, a lo largo del artículo describimos los modos en que se asociaron y organizaron los trabajadores de empresas recuperadas en la Argentina entre los años 2000 y 2015. Identificamos dos períodos diferenciados, tanto respecto al proceso de recuperación de empresas como a las formas en que sus trabajadores se asociaron. En la sociogénesis del proceso, los trabajadores crearon organizaciones de empresas recuperadas que conformaron un movimiento social, y con la estabilización, el carácter movimentista de las organizaciones se fue diluyendo, a la vez que las formas organizativas tendieron a ampliarse dando lugar a una convergencia cooperativa, un acercamiento sindical y un agrupamiento sectorial. Esta ampliación se encontró vinculada tanto con la potencia simbólica de las empresas recuperadas, como con el aporte de las políticas públicas desarrolladas entre los años 2003 y 2015 -principalmente por el Gobierno nacional- que coadyuvaron a conformar el campo de la economía social y popular, en vinculación con el cooperativismo.

Ahora bien, en un nuevo contexto social, político y económico inaugurado no sólo con el cambio de gobierno del Estado nacional 
a fines del año 2015, sino también con las transformaciones políticas regresivas operadas a nivel regional, nos preguntamos acerca de los posibles cambios en las formas de organización y asociación del universo social indagado, así como sobre viabilidad de estas formas socioproductivas, en el marco de una estructura distinta de oportunidades $-\mathrm{o}$ restricciones- para la acción colectiva y de cambios en el modelo de acumulación. En un contexto de estancamiento económico y de condiciones adversas al mercado interno: ¿En qué medida tienen lugar nuevas recuperaciones? ¿Y sobre qué elementos se asientan sus desenvolvimientos posteriores? ¿Cómo se reacomodan los proyectos político-productivos de las empresas recuperadas en un contexto político y económico desfavorable a su desarrollo? ¿Cuáles son las nuevas demandas y las estrategias para plantearlas? ¿Continuarán teniendo relevancia los clusters sectoriales? Tomando en consideración el señalamiento de Wright (2015) acerca de que las limitaciones que imponen las bajas escalas y el aislamiento de las experiencias individuales pueden sortearse a través del establecimiento de vínculos y articulaciones que permitan conformar un mercado cooperativo (Wright, 2015), nos preguntamos ¿en qué medida este contexto puede posibilitar u obstaculizar la consolidación de un mercado cooperativo?

En otro orden de cosas, y tal como mencionamos, este cambio de escenario ha dado lugar a la realización de acciones colectivas de gran envergadura protagonizadas por organizaciones de empresas recuperadas y de cooperativas de programas sociales. Incluso, este tipo de acciones lograron la sanción en el año 2016 de la Ley de Emergencia Social ${ }^{15}$ motorizada por la CTEP. Tomando en consideración nuevamente que el incremento del poder social a que dan lugar las formas cooperativas se potencia a partir de su capacidad de desarrollar mayores grados de articulación organizativa entre las experiencias aisladas (Wright, 2015), nos preguntamos, ¿configuran estas acciones colectivas un basamento para la profundización de la convergencia cooperativa señalada? Ahora bien, a la vez que el nuevo escenario ha propiciado las mencionadas acciones, también ha exacerbado los procesos represivos de la protesta social. De este modo, las preguntas planteadas respecto a las formas de organiza- 
ción de los trabajadores sin patrón, generalmente ubicados entre los sectores más excluidos, oprimidos y pauperizados, se evidencian más pertinentes aún en el contexto social y político que plantea la Argentina actual, en tanto eleva las privaciones relativas y azuza la acción de resistencia, a la vez que resulta desfavorable a la acción colectiva beligerante en el espacio público.

\section{REFERENCIAS}

ACTO FUNDACIONAL DE LA CONFEDERACIÓN DE TRABAJADORES DE LA ECONOMÍA POPULAR. (19 de agosto de 2011). La Alameda. In: https://laalameda.wordpress. com/2011/08/19/acto- fundacional-de-la-confederacion-detrabajadores-de-la-economia-popular-ctep/ accedido en 25 jun. 2016.

ARGENTINA. ¿Qué es la CTEP? (s/d). Resumen Latinoamericano. En: http://www.resumenlatinoamericano.org/2015/02/08/argentinaque-es-la-ctep/ accedido en 22 jun. 2016.

ARCIDIÁCONO, P. y BERMÚDEZ, M. A. Clivajes, tensiones y dinámicas del cooperativismo de trabajo bajo programas sociales. El boom de las cooperativas del PROGRAMA INGRESO SOCIAL CON TRABAJO-ARGENTINA TRABAJA. Revista del CESOT, n. 7, pp. 3-36, 2015.

ASOCIACIÓN NACIONAL DE TRABAJADORES AUTOGESTIONADOS (s/d). Documentos ANTA. En: http:// archivo.cta.org.ar/-Documentos-ANTA-html accedido en 10 nov. 2016.

BASUALDO, E. Estudios de historia económica argentina: Desde mediados del siglo XX a la actualidad. Buenos Aires: Siglo Veintiuno Editores, 2013.

CLARÍN: Brukman: falló una gestión oficial y las obreras acampan en la calle. (23 de abril de 2003). In: http://edant.clarin.com/ diario/2003/04/23/p-01401.htm accedido en 25 sept. 2016.

CTEP (s/d). Nosotros. En: http://ctepargentina.org/nosotros/ accedido en 15 jun. 2013.

DAVOLOS, P. y PERELMAN, L. La intervención sindical en las empresas recuperadas. Un estudio de caso. $6^{\circ}$ Congreso Nacional de Estudios del Trabajo de ASET. Buenos Aires, 13-16 de agosto de 2003. 
Las formas de organización y asociación de los trabajadores de empresas recuperadas en la Argentina (2000-2015).

DE LA GARZA TOLEDO, E. Introducción: construcción de la identidad y acción colectiva entre trabajadores no clásicos como problema. In: DE LA GARZA TOLEDO, E. (Coord.). Trabajo no clásico, organización y acción colectiva (pp.11-21). México: Universidad Autónoma Metropolitana-Iztapalapa-Plaza y Valdés Editores, 2011.

DEUX MARZI, M. V. y HINTZE, S. Protección y seguridad social de los trabajadores asociativos autogestionados. In: DANANI, C. y HINTZE, S. (Coords.). Protecciones y desprotecciones (II). Problemas y debates de la seguridad social en la Argentina (pp.311361). Los Polvorines: Universidad Nacional de General Sarmiento, 2014.

ECHAIDE, J. Debate sobre empresas recuperadas. Un aporte desde lo legal, lo jurídico y lo político. Buenos Aires: Ediciones del Instituto Movilizador de Fondos Cooperativos - Centro Cultural de la Cooperación Floreal Gorini, 2004.

ECHAIDE, J. Sobre lo político y lo jurídico. Lo pendiente en la batalla legal de las empresas recuperadas. In: GARCÍA, A. T. (Coord.). Repensando la economía social (pp.113-144). Buenos Aires: Ediciones del Centro Cultural de la Cooperación Floreal Gorini, 2010.

FAJN, G. Fábricas y empresas recuperadas. Protesta social, autogestión y rupturas en la subjetividad. Buenos Aires: Ediciones del Centro Cultural de la Cooperación Floreal Gorini, 2003.

FARINETTI, M. ¿Qué queda del "movimiento obrero"? Las formas del reclamo laboral en la nueva democracia argentina. Trabajo y Sociedad, n¹, 1999.

GROISMAN, F. Inestabilidad de ingresos y desigualdad durante la reciente fase de recuperación económica en la Argentina (2004-2007). Estudios del Trabajo, n 36, pp. 29-47, 2008.

JORNADA POR EL SALARIO Y EL TRABAJO. Fotos (11 de junio de 2003). Indymedia. En: http://argentina.indymedia.org/ news/2003/06/116265_comment.php accedido en 15 may. 2016.

KASPARIAN, D. Informe: Presentación de la Unión Productiva de Empresas Autogestionadas (UPEA). Revista del OSERA, $\mathrm{n}^{\circ}$ 4, 2010.

KASPARIAN, D. Lucha ¿sin patrón? Un estudio sobre la configuración de la conflictividad de trabajo en empresas recuperadas y cooperativas del Programa Argentina Trabaja. 2017. Tesis (Doctorado en Ciencias Sociales). Facultad de Ciencias Sociales, Universidad de Buenos Aires, Buenos Aires, Argentina. 
KASPARIAN, D. y HERNÁNDEZ, C. Informe: Aunando cooperación: La Federación Red Gráfica Cooperativa. Revista del OSERA, ${ }^{\circ} 4$, 2010.

KASPARIAN, D. y HERNÁNDEZ, C. Informe sobre la Charla-debate: Las empresas recuperadas. Balances y perspectivas. Revista del OSERA, $\mathrm{n}^{\circ} 7,2012$.

MALDOVAN BONELLI, J. La economía popular: debate conceptual de un campo en construcción. Buenos Aires: Universidad Metropolitana para la Educación y el Trabajo, 2018.

MINISTERIO DE DESARROLLO SOCIAL DE LA NACIÓN. Programa de Ingreso Social con Trabajo. Síntesis de resultados e impactos, 2015.

MOVIMIENTO NACIONAL DE FÁBRICAS RECUPERADAS POR SUS TRABAJADORES (s/d). Listado de cooperativas. En: http:// fabricasrecuperadas.com.ar/noticias-movimiento-nacionalfabricas-recuperadas.php?codigo $=4622 \#$.WCN-J_nhDIU) accedido en 10 sept. 2016.

PALOMINO, H. El movimiento de trabajadores de empresas recuperadas. Revista Sociedad, n²0-21, pp. 125-146, 2003.

PALOMINO, H. Los sindicatos y los movimientos sociales emergentes del colapso neoliberal en Argentina. In: DE LA GARZA TOLEDO, E. (Comp.). Sindicatos y nuevos movimientos sociales en América Latina (pp. 19-52). Buenos Aires: CLACSO, 2005.

PALOMINO, H.; BLEYNAT, I.; GARRO, S. y GIACOMUZZI, C. Cuestiones actuales sobre el universo de empresas recuperadas y las nuevas lógicas de agregación de los actores. Revista del OSERA, $\mathrm{n}^{\circ} 5,2011$.

PROGRAMA FACULTAD ABIERTA. Datos preliminares del informe de situación de las empresas recuperadas por los trabajadores a fines de 2017. Buenos Aires: Facultad de Filosofía y Letras - Universidad de Buenos Aires, 2017.

REBÓN, J. La empresa de la autonomía. Trabajadores recuperando la producción. Buenos Aires: Colectivo Ediciones-Ediciones PICASO, 2007.

REBÓN, J. y KASPARIAN, D. La valoración social de las cooperativas en el Área Metropolitana de Buenos Aires. Una aproximación a partir de la investigación por encuesta. Cayapa. Revista Venezolana de Economía Social, n² 29, pp. 11-37, 2015. 
Las formas de organización y asociación de los trabajadores de empresas recuperadas en la Argentina (2000-2015).

REBÓN, J.; KASPARIAN, D. y HERNÁNDEZ, C. La economía moral del trabajo. La legitimidad social de las empresas recuperadas. Trabajo y Sociedad, n²5, pp. 173-194, 2015.

RECONOCIMIENTO PARA LOS TRABAJADORES DE LA ECONOMÍA POPULAR. (14 de diciembre de 2015). Info Gremiales. In: http:// www.infogremiales.com.ar/reconocimiento-para-los- trabajadoresde-economia-popular/ accedido en 25 jun. 2016.

RUGGERI, A. Informe del III Relevamiento de Empresas Recuperadas en la Argentina. Las empresas recuperadas en la Argentina. 2010. Buenos Aires: Facultad de Filosofía y Letras - Universidad de Buenos Aires, 2010.

SALGADO, R. Los límites de la igualdad. Cambio y reproducción social en el proceso de recuperación de empresas por sus trabajadores. 2012. Tesis (Doctorado en Ciencias Sociales). Facultad de Ciencias Sociales, Universidad de Buenos Aires, Buenos Aires, Argentina.

SARRIA ICAZA, A. M. y TIRIBIA, L. Economía popular. In: CATTANI, A. D. (Ed.). La otra economía (pp. 441). Buenos Aires: Editorial Altamira/ Universidad Nacional de General Sarmiento, 2004.

SENÉN GONZÁLEZ, C. y HAIDAR, J. Los debates acerca de la "revitalización sindical" y su aplicación en el análisis sectorial en Argentina. Revista Latinoamericana de Estudios del Trabajo, 2 a Época, no 22, pp. 5-31, 2009.

SOUSA SANTOS, B. y RODRÍGUEZ, C. Para ampliar el canon de la producción. In: SOUSA SANTOS, B. (Coord.). Producir para vivir: los caminos de la producción no capitalista (pp. 15-61). México: Fondo de Cultura Económica, 2011.

TILLY, CH. y WOOD, L. Los movimientos sociales 1768-2009. Madrid: Editorial Crítica, 2010.

Urgente: Represión policial en Movilización en Esteban Echeverría por Cooperativas sin punteros (20 de noviembre de 2009). ANRed. In: http://www.anred.org/spip.php?breve4492 accedido en 20 jul. 2015.

VÁZQUEZ, G. ¿Son sostenibles los emprendimientos asociativos de trabajadores autogestionados? Algunas reflexiones a contramano del sentido común. Voces en el Fénix, n 37, pp. 130-137, 2014.

WILLIAMS, M. The solidarity economy and social transformation. In: SATGAR, V. (Comp.). The solidarity economy alternative: emerging theory and practice (pp.37-63). Durban: University of KwaZulu-Natal Press, 2014. 
WRIGHT, E. O. Construyendo utopías reales. Buenos Aires: Ediciones Akal, 2015.

WYCZYKIER, G. Sobre procesos de autogestión y recolectivización laboral en la Argentina actual. Polis, Revista de la Universidad Bolivariana, Vol. 8, nº 24, pp. 197-220, 2009.

NORMATIVA:

LEY N $N^{\circ} 20.337$ DE COOPERATIVAS. Año 1973. In: http://servicios. infoleg.gob.ar/infolegInternet/anexos/15000-19999/18462/texact. htm accedido en 20 jun. 2016.

RESOLUCIÓN No 784/92 DE LA ADMINISTRACIÓN NACIONAL DE LA SEGURIDAD SOCIAL (ANSES). Año 1992. In: https://www. boletinoficial.gob.ar/\#!DetalleNormaBusquedaAvanzada/7129487/ null accedido en 20 jun. 2016.

RESOLUCIÓN No 183/92 DEL INSTITUTO NACIONAL DE ACCIÓN COOPERATIVA (INAC). Año 1992. In: http://www.inaes.gob.ar/ files/resoluciones/inac/rinac183_92.pdf accedido en 20 jun. 2016.

RESOLUCIÓN $\mathrm{N}^{\circ}$ 4.664/13 DEL INSTITUTO NACIONAL DE ASOCIATIVISMO Y ECONOMÍA SOCIAL (INAES). Año 2013. In: http://www.inaes.gob.ar/files/resoluciones/INAES/ RESOLUCION $\% 20 \% 20 \% 20 \% 20 \% 20 \% 20 \quad$ N\% $204664-13 \% 20$ INAES.pdf accedido en 20 jun. 2016.

\section{ENTREVISTAS CITADAS:}

Entrevista Autoridad de FECOOTRA, mayo 2013.

Entrevista Referente del movimiento de empresas recuperadas, septiembre 2016. 